\title{
Positron Annihilation in Carbon Nanotubes Studied by Coincidence Doppler Broadening Spectroscopy
}

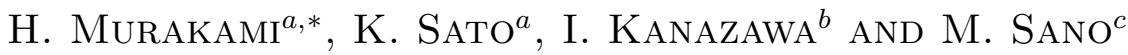

${ }^{a}$ Department of Environmental Science, Faculty of Education

Tokyo Gakugei University, Koganei, Tokyo 184-8501, Japan

${ }^{b}$ Department of Physics, Faculty of Education, Tokyo Gakugei University

Koganei, Tokyo 184-8501, Japan

${ }^{c}$ Department of Chemistry, Division of Natural Sciences International Christian University, Mitaka, Tokyo 181-8585, Japan

In order to assign the sites of positron annihilation, coincidence Doppler broadening spectra were measured for a highly oriented pyrolytic graphite crystal, graphite powder, multi-walled carbon nanotubes (MNTs) and cupstacked carbon nanotubes (CNTs). The spectrum for graphite powder normalized to that for highly oriented pyrolytic graphite (HOPG) is almost flat in the momentum region from $7 \times 10^{-3}$ to $13 \times 10^{-3} m_{\mathrm{e}} c$, having a ratio close to unity. The flat spectrum demonstrates that positrons injected into graphite powder annihilate in the interlayer spaces of piled graphite sheets, in the same manner as positrons in highly oriented pyrolytic graphite annihilate in the bulk. The coincidence Doppler broadening spectra for MNTs and CNTs are quite different from that for highly oriented pyrolytic graphite, which indicates that positrons injected into MNTs and CYTs annihilate not in the bulk, but on surface. The positron lifetime spectrum for multi-walled carbon nanotubes is analyzed in terms of a single component due to surfacetrapped positrons, while that for CNTs is decomposed into three components attributable to para-positronium surface-trapped positrons and orthopositronium. The difference between the coincidence Doppler broadening spectrum for CNTs and that for MNTs is explained in terms of positron annihilation on zigzag surfaces of CNTs which are composed of both graphitesheet and graphite-edge planes.

PACS numbers: $61.46 . \mathrm{Fg}, 78.70 . \mathrm{Bj}, 36.10 . \mathrm{Dr}$

\section{Introduction}

Graphite is a two-dimensional material composed of graphite sheets (hexagonal carbon networks) piling up along the $c$-axis with a gap between two adjacent

${ }^{*}$ corresponding author; e-mail: murakami@u-gakugei.ac.jp 
sheets of $0.335 \mathrm{~nm}$. Recently, carbon materials different from graphite in morphology were prepared: MNTs [1] and CNTs [2]. A single MNT consists of concentric microtubes of graphite sheet, with several tens of nanometers in diameter and ca. $1 \mu \mathrm{m}$ in length. A single CNT, ca. $50 \mathrm{~nm}$ in outer diameter and $c a .25 \mu \mathrm{m}$ in length, comprises stacked cups made of 9-12 truncated conical graphite sheets with an interlayer spacing of $c a .0 .34 \mathrm{~nm}$. It has a long hollow core around the tube axis, $c a .15 \mathrm{~nm}$ in diameter. The graphite sheets incline at $c a .20^{\circ}$ with respect to the axis, so that zigzag outer- as well as inner-surfaces of the tubes consist of graphite-sheet planes (forming bodies of cups) and graphite-edge planes (forming brims of cups).

The positron lifetime spectrum for a highly oriented pyrolytic graphite (HOPG) crystal consists of two components: $0.210 \mathrm{~ns}(91 \%)$ and $0.390 \mathrm{~ns}(9 \%)$ [3]. The short lifetime observed for graphite has been ascribed to free positrons and the longer one to surface-trapped positrons [4]. Positron lifetime spectra for pristine MNTs and alkali metal-doped MNTs consist of a single component, their lifetimes being almost the same in the range of $0.36 \mathrm{~ns}$ [5]. The positron lifetime spectrum for CNTs is decomposed into three components; $0.125 \mathrm{~ns}$ (fixed) (6\%), $0.345 \mathrm{~ns}$ $(75 \%)$ and $1.21 \mathrm{~ns}(19 \%)$. The shortest-lived component is attributed to the annihilation of para-positronium ( $p$-Ps) and the longest-lived one to that of ortho-Ps (o-Ps). The yield of Ps atoms is found to be quite high, 25\%, compared with those for other graphite materials [6].

Here we studied the annihilation of positrons in an HOPG crystal, graphite powder (GP), MNTs and CNTs by coincidence Doppler broadening (CDB) spectroscopy in order to assign the sites of positron annihilation.

\section{Experimental}

CDB spectra were obtained with the aid of a 2-parameter coincidence-measurement system (NT24-DUAL 2 parameter, Laboratory Equipment Co.) of two Ge detectors arranged at $180^{\circ}$ to each other. A sample including a positron source at its center was placed at the optimum position on a line between the two detectors to achieve simultaneously a high energy resolution of $c a .1 .1 \mathrm{keV}$ and a high count-rate of $c a .700 \mathrm{cps}$ for a source-detector distance of $20 \mathrm{~cm}$. A coincidence time was set at $1 \mu \mathrm{s}$.

Samples of MNTs (BU-201, Bucky Co.) and CNTs (Carbere 24HT, GSI Creos Co.) for CDB measurements were prepared by being accommodated in pyrex-glass ampoules which contained a ${ }^{22} \mathrm{NaCl}$ positron source of $740 \mathrm{kBq}$ sealed between two kapton films of $7.5 \mu \mathrm{m}$ thick. Carbon nanotubes surrounded the positron source entirely. Two pieces of HOPG $\left(10 \times 10 \times 1.5 \mathrm{~mm}^{3}\right.$, GRAS/1.5, ZYA, NT-MDT Co.), facing with their $a b$ planes, sandwiched a positron source of $740 \mathrm{kBq}$. CDB spectra were measured in two directions; in parallel and perpendicular to the $a b$ plane. A sample of GP plates $\left(10 \times 10 \times 1 \mathrm{~mm}^{3}\right.$, G530S, Tokai Carbon Co.) was prepared in the same manner as HOPG. 


\section{Results and discussion}

Figure 1 shows a two-dimensional CDB display for CNTs obtained from $1.2 \times 10^{6}$ events. Coincident numbers of $\gamma$ rays are plotted as a function of their energies, $E_{1}$ and $E_{2}$, along the horizontal and vertical axes. Each axis is $48 \mathrm{keV}$ wide. There is an intense central peak at $E_{1}=E_{2}=511 \mathrm{keV}$, with accumulated counts of $c a .4 \times 10^{4}$. The elliptical region extending diagonally is nearly free from a background. Spectra shown in Fig. 2 were obtained from a projection of the display onto $E_{1}-E_{2}=P_{\mathrm{L}} c$ axis, where $P_{\mathrm{L}}$ and $c$ are a longitudinal component of an electron-positron momentum along the direction of $\gamma$ ray measurement and the speed of light, respectively. The projected spectra include events observed through

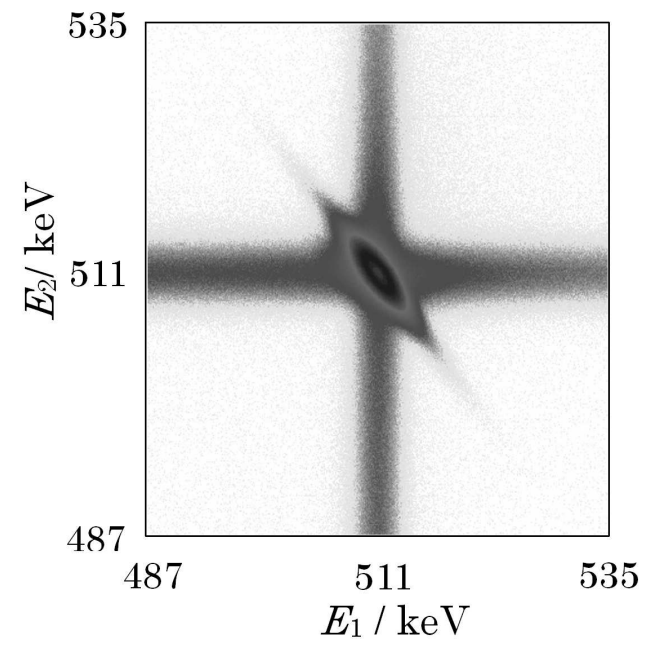

Fig. 1. A two-dimensional display of coincident event in CNTs.

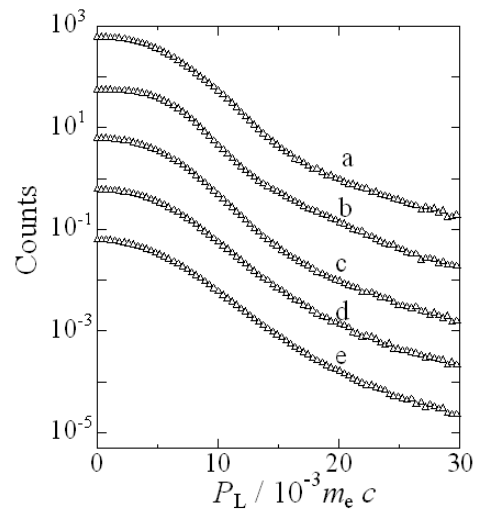

Fig. 2. CDB spectra obtained for $a-\mathrm{HOPG}, \gamma \| a b, b-\mathrm{HOPG}, \gamma \perp a b, c-\mathrm{GP}, d$ - CNTs and $e-$ MNTs. 
a window along the diagonal with $2 m_{\mathrm{e}} c^{2}-1.9 \mathrm{keV}<E_{1}+E_{2}<2 m_{\mathrm{e}} c^{2}+1.9 \mathrm{keV}$, where $m_{\mathrm{e}}$ is the electron rest mass. All spectra are normalized to unity in area.

The CDB spectra thus obtained are normalized to the CDB spectrum for HOPG obtained by averaging the CDB spectrum observed in parallel to the $a b$ plane and that observed in perpendicular to the plane, as shown in Fig. 3. The normalized spectrum for GP is almost flat in the momentum region from $7 \times 10^{-3}$ to $13 \times 10^{-3} m_{\mathrm{e}} c$, having a ratio close to unity. The flat spectrum indicates that the Doppler broadening spectra are derived from the same functional formula for both HOPG and GP, and positrons injected into GP annihilate in the bulk of piled graphite sheets.

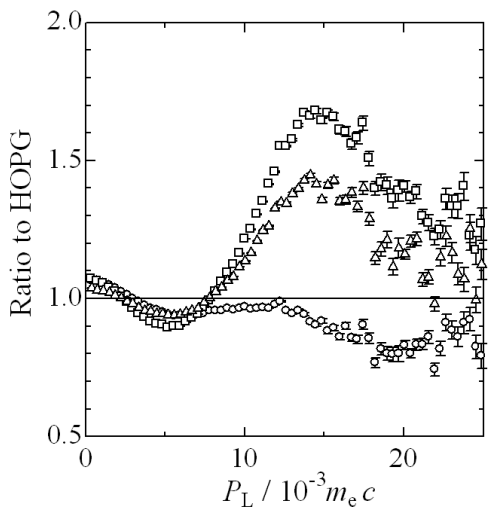

Fig. 3. Spectra for GP $(\bigcirc)$, MNTs $(\triangle)$ and CNTs $(\square)$ normalized to HOPG.

The normalized spectra for MNTs and CNTs change in their height with an increase in $P_{\mathrm{L}}$ up to $15 \times 10^{-3} m_{\mathrm{e}} c$, and are indefinite in the region higher than $15 \times 10^{-3} m_{\mathrm{e}} c$, owing to statistical uncertainty. The uneven shapes of the spectra in the entire region indicate that positrons injected into MNTs and CNTs annihilate not in the bulk, but on surface.

The positron lifetime spectrum for MNTs has been analyzed in terms of a single component due to surface-trapped positrons [5], while that for CNTs has been analyzed with three components attributable to $p$-Ps, surface-trapped positrons and $o$-Ps. $p$-Ps atoms are formed in CNTs with a yield of $6 \%$ [6].

The entire Doppler broadening spectrum for CNTs, $f^{\mathrm{C}}\left(P_{\mathrm{L}}\right)$ is expressed as follows:

$$
f^{\mathrm{C}}\left(P_{\mathrm{L}}\right)=0.94 f^{\mathrm{CO}}\left(P_{\mathrm{L}}\right)+0.06 f^{\mathrm{p}-\mathrm{Ps}}\left(P_{\mathrm{L}}\right)
$$

where $f^{\mathrm{CO}}\left(P_{\mathrm{L}}\right)$ and $f^{\mathrm{p}-\mathrm{Ps}}\left(P_{\mathrm{L}}\right)$ are a Doppler broadening spectrum not including a contribution from $p$-Ps annihilation and that originating from $p$-Ps annihilation, respectively. Each spectrum is normalized as follows: 


$$
\int_{0}^{\infty} f^{\mathrm{C}}\left(P_{\mathrm{L}}\right) \mathrm{d} P_{\mathrm{L}}=\int_{0}^{\infty} f^{\mathrm{CO}}\left(P_{\mathrm{L}}\right) \mathrm{d} P_{\mathrm{L}}=\int_{0}^{\infty} f^{\mathrm{p}-\mathrm{Ps}}\left(P_{\mathrm{L}}\right) \mathrm{d} P_{\mathrm{L}}=1 .
$$

A spectrum of difference between $f^{\mathrm{C}}\left(P_{\mathrm{L}}\right) / 0.94$ and $f^{\mathrm{M}}\left(P_{\mathrm{L}}\right), D\left(P_{\mathrm{L}}\right)$ is expressed as

$$
\begin{aligned}
D\left(P_{\mathrm{L}}\right) & =f^{\mathrm{C}}\left(P_{\mathrm{L}}\right) / 0.94-f^{\mathrm{M}}\left(P_{\mathrm{L}}\right) \\
= & {\left[f^{\mathrm{CO}}\left(P_{\mathrm{L}}\right)-f^{\mathrm{M}}\left(P_{\mathrm{L}}\right)\right]+(0.06 / 0.94) f^{\mathrm{p}-\mathrm{Ps}}\left(P_{\mathrm{L}}\right), }
\end{aligned}
$$

where $f^{\mathrm{M}}\left(P_{\mathrm{L}}\right)$ is a Doppler broadening spectrum for MNTs. $D\left(P_{\mathrm{L}}\right)$ is obtained from the experimentally observed spectra, $f^{\mathrm{C}}\left(P_{\mathrm{L}}\right)$ and $f^{\mathrm{M}}\left(P_{\mathrm{L}}\right)$, as shown in Fig. 4.

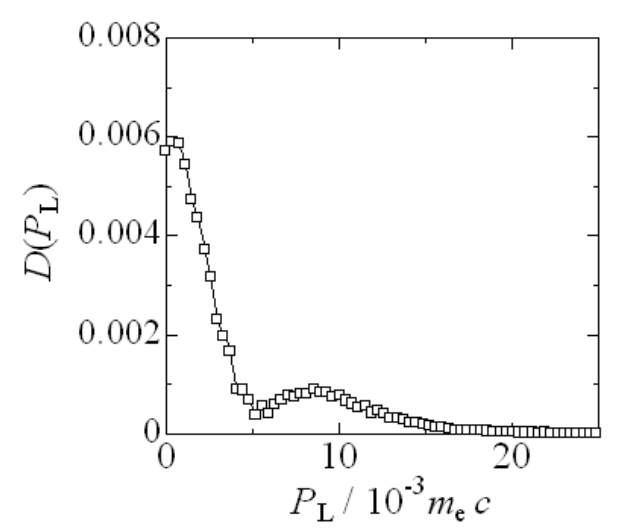

Fig. 4. Difference spectrum, $D\left(P_{\mathrm{L}}\right)$.

$D\left(P_{\mathrm{L}}\right)$ is found to consist of two bands; a narrow band decreasing to zero at ca. $5 \times 10^{-3} m_{\mathrm{e}} c$ and a broad one with a long tail up to $15 \times 10^{-3} m_{\mathrm{e}} c$. The spectrum $f^{\mathrm{p}-\mathrm{Ps}}\left(P_{\mathrm{L}}\right)$ derived from $p$-Ps annihilation has been reported to decrease steeply in height to zero at $c a .2 \times 10^{-3} m_{\mathrm{e}} c$ with an increase in $P_{\mathrm{L}}$ [7], so that the narrow band observed is attributed to the annihilation of $p$-Ps. The broad band indicates that CNTs possess positron-annihilation sites different in character from those of MNTs. A surface of an MNT is formed only with graphite-sheet planes, while a surface of a CNT is composed of both graphite-sheet and graphite-edge planes, so that the positron annihilation which is observed as a broad band on $D\left(P_{\mathrm{L}}\right)$ is explained in terms of the annihilation of positrons at the sites on graphite-edge planes. Ps atoms are also formed on graphite-edge planes $[6,8]$.

\section{Acknowledgments}

This work was partially supported by Grant-in-Aid for Scientific Research of the Ministry of Education, Culture, Sports, Science and Technology (grant No. 18560651). 


\section{References}

[1] S. Iijima, Nature 354, 56 (1991).

[2] M. Endo, Y.A. Kim, T. Hayashi, Y. Fukai, K. Oshida, M. Terrones, T. Yanagisawa, S. Higaki, M.S. Dresselhaus, Appl. Phys. Lett. 80, 1267 (2002).

[3] H. Murakami, I. Kanazawa, M. Sano, T. Enoki, H. Inokuchi, Synth. Met. 32, 135 (1989).

[4] Y.C. Jean, K. Venkateswaran, E. Parsai, K.L. Cheng, Appl. Phys. A 35, 169 (1984).

[5] H. Murakami, M. Sano, J. Phys. Soc. Jpn. 71, 125 (2002).

[6] H. Murakami, T. Hiejima, M. Sano, Mater. Sci. Forum 445-446, 331 (2004).

[7] P. Sferlazzo, S. Berko, K.G. Lynn, A.P. Mills, Jr., L.O. Roellig, A.J. Viescas, R.N. West, Phys. Rev. Lett. 60, 538 (1988).

[8] H. Murakami, T. Hiejima, M. Sano, J. Phys. Soc. Jpn. 74, 1320 (2005). 